\title{
Optimizing Case-based Algorithm for Maintaining the Photovoltaic Systems
}

\author{
A.H. Mohamed \\ Solid State and Electronic Accelerators Dept. \\ National Centre for Radiation Research \& Technology (NCRRT) \\ Atomic Energy Authority
}

\begin{abstract}
Maintenance of the photovoltaic (PV) systems is a very important task for improving their performance. Many parameters affect the PV operation such as the environmental conditions, panel's size and direction. These nonlinear parameters cause NP-hard problem that is represented as a very complex task to be solved. Researchers have developed many traditional systems to deal with these problems. But, there is a little work concerns the automation of the event maintenance appeared at the trouble environmental conditions. The proposed system introduces a new intelligent case based system that can use the fuzzy logic representation to deal with the uncertain and unknown values represented the case in the case library of the PV systems. Besides, the case based system uses the genetic algorithm optimization technique to overcome the complexity of the adaptation problem. The proposed system is a general purpose system that can be used in different CBR systems. It has been applied for different event maintenance process of a PV system. Its obtained results have proved its good performance compared to present methods for the maintenance process of the PV systems.
\end{abstract}

\section{Keywords}

Case based reasoning, Fuzzy logic, Genetic algorithm, Photovoltaic system, and Maintenance process.

\section{INTRODUCTION}

Environmental conditions can affect mainly the operation of the PV systems and their output power. Therefore, the maintenance process of the PV systems is a main task for ensuring its cleaning, adjusting,... and so their good performance $[1,2]$. But, automating this maintenance process is a very complex task.

Many systems have been developed to manage the maintenance process of the PV systems based on their schedule [3-7]. But, there is little work developed to solve the problems when the PV systems face trouble environmental conditions. Besides, they still face some limitations due to their complexity and cost.

However, the proposed system introduces the uses of fuzzycase based reasoning and the genetic algorithm to automate the maintenance operation of the PV systems. It uses the fuzzy representation into the case based system to manage the uncertain values appeared in the practical situations. Besides, it suggests improving its performance by incorporating genetic algorithm technique for optimizing the adaptation process. Suggested intelligent optimized case based system can be applied for solving many different problems. It has been applied to improve the performance of the maintenance process of the PV systems.

The reminder of this paper can be organized as: Section 2 represents the case based reasoning systems. Section 3 introduces the fuzzy logic representation. Section 4 gives a brief overview of the genetic algorithms. Section 5 deals with the proposed system, its applicability and results. While, section 6 presents the conclusion.

\section{CASE-BASED REASONING}

Case-Based Reasoning (CBR) is a method of solving current problem by using the solutions of the previous similar problems. A CBR system draws its knowledge from a reasonably large set of cases contained in the case library [8].

The process of CBR is generally composed of five phases as given in Fig. (1): presentation, retrieve, reuse (adaptation), revise (validation) and retrain (update).

1. Representation: represent the cases (problems and their corresponding solutions) stored in the case library. Cases must be identified in such a way to be compatible with the index and retrieval methods.

2. Retrieve: Find the most similar cases to the target problem.

3. Reuse: Adapt the solutions of the past cases to solve the new problem. The adaptation process is the core of the CBR systems. It can generate a solution for the new problem using the past cases. There are many different adaptation techniques used in case-based reasoning such as substitution adaptation, transformational adaptation, derivational (generative) adaptation, and compositional adaptation are mentionable [9].

4. Revise: Revise the proposed solution for the new problem and verify it against the case base.

5. Retain: Retain the learned case (solved problem) in the form of the stored cases to the case base for future problem solving [10].

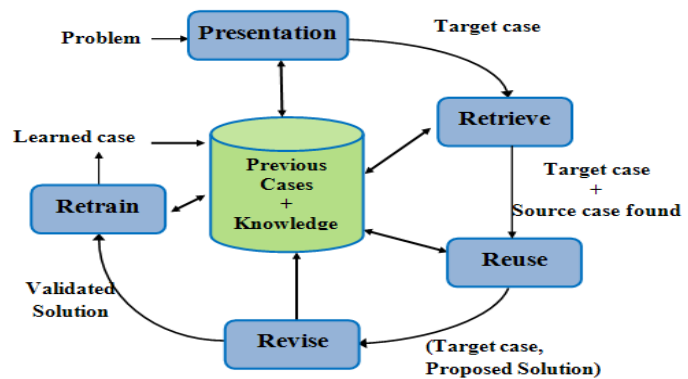

Fig. 1 Case Base Reasoning Cycle

\section{FUZZY LOGIC REPRESENTATION}

Fuzzy logic representation is a modeling method that enables the systems to apply approximate reasoning rather than exact values. Fuzzy logic (FL) provides a simple way to achieve a conclusion based upon vague, ambiguous, imprecise, noisy, or 
missing input information. So, FL approach can solve problems much faster than the traditional ways [11].

It incorporates a simple IF-THEN rule-based approach to solve the problems rather than modeling a system mathematically. The FL model is empirically-based and depending on the developer's experience [12]. For example, rather than dealing with temperature control in terms such as "T < 1000F", or "210C < TEMP <220C", or "IF (process is too hot) AND (process is heating rapidly) THEN (cool the process quickly)" are used [13]. These terms are imprecise and yet critically descriptive of what must actually happen. But, they enable the FL to conclude the results at very high rate [14].

\section{GENETIC ALGORITHMS}

Genetic Algorithms (GAs) are biologically inspired and have a great success in scientific and engineering optimization or search problems. They allow evaluating many parallel schemas at once $[15,16]$. GAs based on the natural selection of the fittest individual for the production of the new generation. In GA the solution of the problem is encoded as the chromosome of the biological system. It keeps a population of randomly selected chromosomes to combine by mutation or crossover techniques and produce the offspring having new characteristics, which replaces the low fitness old chromosomes. This process is repeated until we find a chromosome with best characteristics for the successive generations of the population. Finally, the GA tends to converge on an optimal or near-optimal solution through these operators [17, 18]. Figure (2) illustrate an overview of the proposed GA operation.

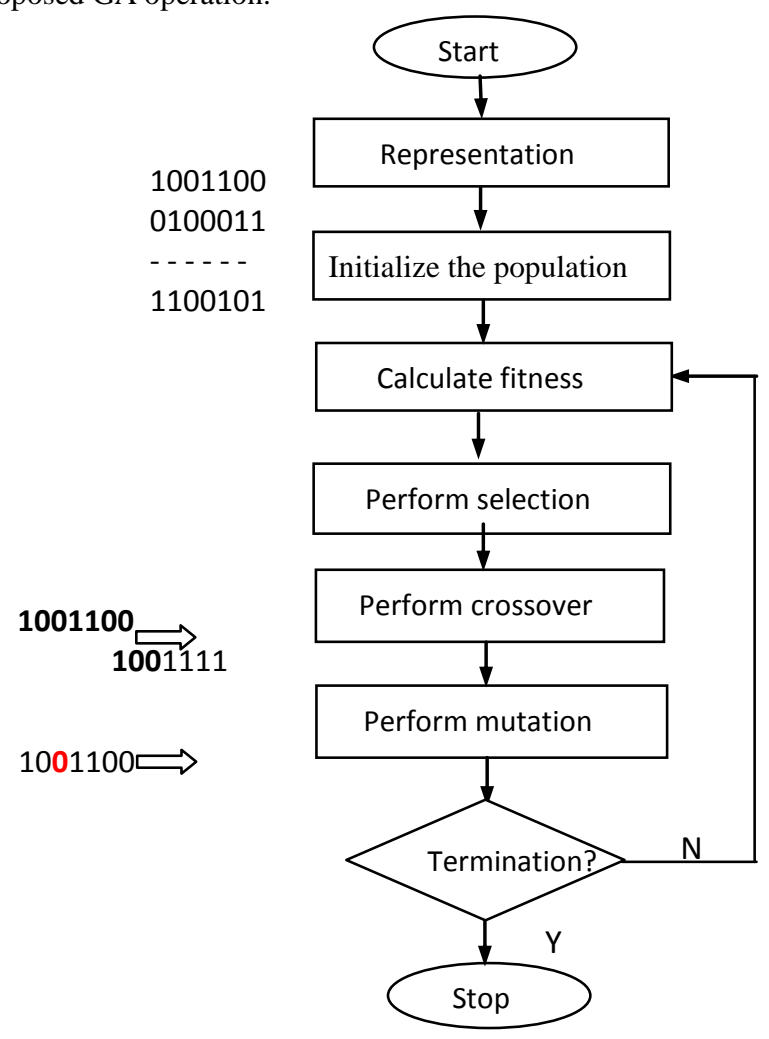

Fig. 2 Basic steps of GA operation

\section{PROPOSED SYSTEM}

The basic idea behind the proposed system is introducing an intelligent system that can optimize the case based reasoning. It uses the fuzzy representation to deal with the uncertain and unknown values of the parameters. It has been developed to improve the maintenance management of the PV systems.
The proposed system divides the operation of the maintenance process of the PV panel into two main phases as shown in fig. (3).

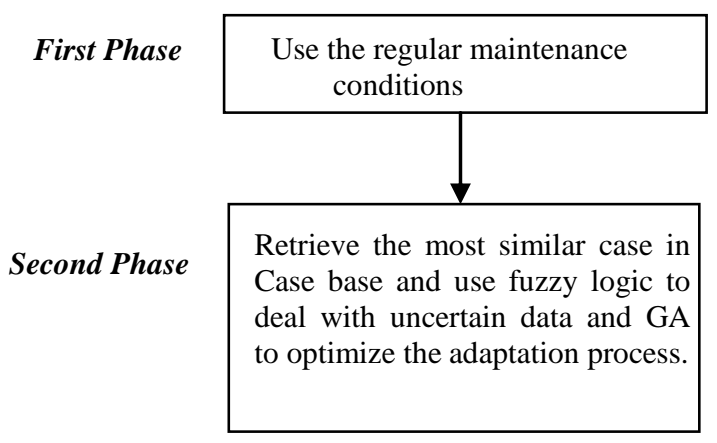

Fig. 3 Main Modules of the Proposed System

The first one deals with the regular maintenance process applied for the PV system based on its previous prepared maintenance schedule. While, the second phase is the events maintenance module that applied only when the PV system faces untraditional events.

\section{- First Phase: Regular Maintenance schedule}

For the regular maintenance of the PV system uses the preprepared steps of the maintenance. These steps are applied according to the time schedule that can be varied based on the natural of the environment around the PV system, its applications, situations, expected changes in the surrounded environment, etc.

However, during the regular maintenance, the system can maintain the solar panel, inverter, AC disconnect and service panel as:

- Regular Maintenance steps for Solar Array (panel) has the following Maintenance Activity:

1. Clean Panel Module.

2. Check all the cables to ensure the good connections and avoid any mechanical damage.

3. Check mechanical security of the array structure of the panel.

4. Check the output voltage and current of each parallel solar array and compare them to the expected output under the same conditions.

5. Check for shading by any surrounded environments such as trees and houses around modules if found.

- While, the maintenance steps for the inverter, AC disconnect and service panel has the following Maintenance activity steps as:

1. Check if all the units have not been affected by spiders, rodents or insects.

2. Cleaning all units to remove dust build Up.

3. Check that all electrical connections are clean and tight.

- Second Phase: Event Maintenance Module

When the system's sensors record un-normal environmental conditions such as high rate or danger level of dust, clouds, windings, .. etc. This drives the system to apply an un-regular maintenance process. In this case, the proposed system uses 
the case based reasoning module to retrieve the most similar case(s) from the case library. Then, it applies the adaptation process to find the required maintenance method (needed solution).

On the other side, the fuzzy logic can deal with the uncertain and unknown data in the cases that mostly appeared in the event of the trouble environmental conditions.

The proposed fuzzy representation describes the system as fuzzy sets. A fuzzy set $A$ in $X$ is characterized by its membership function $\mu \mathrm{A}: \mathrm{X}_{-}[0,1]$ and $\mu \mathrm{A}(\mathrm{x})$ is interpreted as the degree of membership of element $\mathrm{x}$ in fuzzy set A for each $x_{-} X$, where, $X$ is a nonempty set [19].

The proposed fuzzy inference system consists of the following four basic components as shown in fig. (4):

- Fuzzification module,

- A rule-base with if-then rules and related membership functions,

- An inference engine applying algorithms on the rule base and

- A defuzzification module [20].

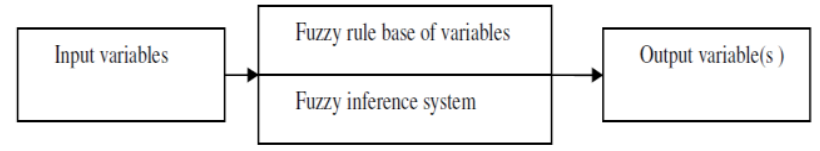

Fig. 4 General Architecture of a Fuzzy Inference System

On the other hand, the CBR module uses the genetic algorithm to optimize the important features affect the solution for the problem at hand. Thus, for each event, there is some different parameters are more important based on the present event conditions.

The proposed system uses the derivational (generative) adaptation technique. It uses the genetic algorithm technique to optimize the parameters used in this adaptation method.

However, the operation of CBR module of the proposed system is represented as follows:

1. The system gets the features represent the problem at hand.

2. The system retrieves the most suitable case(s) from the case library. It uses the fuzzy logic representation to face the uncertain or unknown values of the data for similar case in the library.

3. The system optimizes the features of the retrieved case(s) used in the adaptation task. Thus, the genetic algorithm can optimize the important features that affect the solution for the present problem.

4. The adaptation process uses the optimized features only. Therefore, the adaptation task can be simplified and its time and complexity are decreased.

5. Then, the system retains the new problem and its solution (the required maintenance method suitable for the present problem) for future uses.
The implementation of the GA system can be represented as:

1. Chromosome Representation: The binary values are used for representing the chromosomes. Each chromosome represents the features of the solution.

2. Initial Population: The initial population is constructed from a group of randomly generated chromosomes. The population size is: 40 .

3. Fitness Evaluation: The offspring's can be evaluated according to the following fitness function as:

$$
\mathrm{F}=\sum_{i=1}^{N}\left[x_{i} y_{i}\right]
$$

where: (x) is the weight of the features, $(y)$ is the features of the maintenance process, (i): an instant of the features and $(\mathrm{N})$ is the total number of features need maintenance.

4. Selection Methodology: The proposed system uses the roulette-wheel selection scheme. It is a form of fitnessproportionate selection method.

5. Crossover: Proposed GA uses a single point crossover in which a point of exchange is set at a random location in the two individuals' genomes. The crossover probability is: 0.75 .

6. Mutation: Flip Bit mutation is applied to the offspring. The mutation rate is: 0.15 .

7. Termination: The maximum generations of GA are: 50 .

\section{EVALUATION OF THE PROPOSED SYSTEM}

To evaluate the performance of the proposed system, it is applied for three different simulated environmental conditions. The most important feature affect the problem for the first simulated one is the heavily raining faces the PV system, while the second is the high rate dusty winding causes removing for some electrical connections. The third one is the thunder and lightning that causes damage for some solar cells. The same conditions are applied for a traditional case based system and a CBR-Fuzzy method. Table (1) shows a comparison between the results obtained from the proposed optimized CBR-Fuzzy system, a traditional CBR system and a CBR-Fuzzy system. It is found that, the proposed system has increased the performance of the PV system rather than the traditional CBR and CBR-Fuzzy systems.

\section{CONCLUSION}

This research suggests a new case based reasoning system that can use the fuzzy logic technique to deal with the uncertain and the unknown values. Also, the proposed system has used the genetic algorithm optimization technique to optimize the important features used in the adaptation task of CBR system for the problem at hand. However, the proposed system can improve its performance due to:

1. It can increase the performance of the case based systems by using the fuzzy logic to be more applicable in practice. 
Table 1: Shows a comparison between the Results Obtained from the Proposed System, Traditional a CBR System and a CBRFuzzy Systems

\begin{tabular}{|c|c|c|c|}
\hline CBR Systems & $\begin{array}{l}\text { No. of retrieved case } \\
\text { bases }\end{array}$ & Average Accuracy & Average Time \\
\hline \multicolumn{4}{|c|}{ Simulated Environmental -1 } \\
\hline $\begin{array}{l}\text { Proposed optimized } \\
\text { CBR-Fuzzy based system }\end{array}$ & 5 & $97.32 \%$ & $4.2 \mathrm{~min}$. \\
\hline CBR-Fuzzy based system & 5 & $89.14 \%$ & $6.4 \mathrm{~min}$. \\
\hline Traditional CBR System & - & $78.41 \%$ & 7.7 min. \\
\hline \multicolumn{4}{|c|}{ Simulated Environmental -2 } \\
\hline $\begin{array}{l}\text { Proposed optimized } \\
\text { CBR-Fuzzy based system }\end{array}$ & 6 & $96.86 \%$ & $4.7 \mathrm{~min}$. \\
\hline CBR-Fuzzy based system & 6 & $896 \%$ & 7.6 min. \\
\hline Traditional CBR System & - & $74.11 \%$ & 8.7 min. \\
\hline \multicolumn{4}{|c|}{ Simulated Environmental -3 } \\
\hline $\begin{array}{l}\text { Proposed optimized } \\
\text { CBR-Fuzzy based system }\end{array}$ & 4 & $97.01 \%$ & $5.0 \mathrm{~min}$. \\
\hline CBR-Fuzzy based system & 6 & $83.4 \%$ & $9.0 \mathrm{~min}$. \\
\hline Traditional CBR System & - & $73.4 \%$ & $10.3 \mathrm{~min}$. \\
\hline
\end{tabular}

2. It can increase the performance of the case based systems by using the fuzzy logic to be more applicable in practice.

3. It can improve the accuracy of the case based system by using the fuzzy logic that enables the system to improve the retrieving process.

4. It can improve the performance of the adaptation process by concerning only on the optimized features for the problem at hand. It drives for decreasing its complexity and time.

5. It is a general purpose optimized CBR-Fuzzy system. It can be applied for solving many different types of problems.

6. It has been applied for a PV system to solve the selecting and using the most suitable maintenance method for the event environmental conditions. It has proven its performance to be applied in practice to solve real problems.

7. It can decrease the human errors may be appeared due to unsuitable maintenance method are used.

\section{REFERENCES}

[1] S. Oshaba, E. S. Ali and S. M. Abd El-azim, (2015), Artificial Bee Colony Algorithm Based Maximum Power Point Tracking in Photovoltaic System, WSEAS TRANSACTIONS on POWER SYSTEMS, Vol. 10, pp.124-134.

[2] M. Izadbakhsh, M. Gandomkar, A. Rezvani, and A. Ahmadi, (2015), "Short-Term Resource Scheduling Of A Renewable Energy Based Micro Grid," Renewable Energy, Vol. 75, pp. 598-606.
[3] A. Aamodt.; E. Plaza, (1994), Case-based reasoning: foundational issues, methodological variations and system approaches. AI Communications, 7(1), pp.39-59.

[4] J. Haney and A. Burstein, (2013), "PV System Operations and Maintenance Fundamentals", Next Phase Inc., http://www.solarabcs.org.

[5] Dave Williams, Large-Scale PV Operations and Maintenance, http://solarprofessional.com/

[6] T.J. Keating, A. Walker and K. Ardani, (2015), SAPC Best Practices in PV Operations and Maintenance, http://www.nrel.gov/publications.

[7] D. Tan, A. K. Seng, Handbook for Solar Photovoltaic (PV) Systems, http://www.bca.gov.sg/

[8] Australian Business Council for Sustainable Energy, Solar PV Systems Users' Maintenance Guide, http:// www.bcse.org.au

[9] A. Cordier, B. Mascret, A. Mille, (2013), "Dynamic Case Based Reasoning for Contextual Reuse of Experience", Case-Based Reasoning Workshop, ICCBR 2010, Cindy Marling ed. Alessandria, Italy, pp. 69-78.

[10] E. Plaza and L. Mcginty, (2013), "Distributed case-based reasoning, The Knowledge Engineering" Review, Vol. 72. No.4, Cambridge University Press, pp. 30-37.

[11] H. Bustince, J. Montero, M. Pagola, E. Barrenechea and D. Gomez, (2008), "A survey of Interval-Valued Fuzzy Sets", In: Handbook of Granular Computing, Addison Wesley, pp. $491-516$.

[12] H. Guo, Yi Feng, F. Hao, S. Zhong, S. Li., (2014), Dynamic Fuzzy Logic Control of Genetic Algorithm Probabilities, Journal of Computers, VOL. 9(1): pp.2227. 
[13] A. Evsukoff, S. Gentil and J. Montmain, (2000), Fuzzy Reasoning in Cooperative Supervision Systems, Control Engineering Practice, pp. 389- 407.

[14] I. Fliss and M.Tagina, (2010), Multiple Faults Fuzzy Detection Approach, International Conference of Modeling and Simulation- MOSIM'10, Hammamet, Tunisia, May 10-12, pp.7-13.

[15] M. Patrascu; A.F. Stancu; F. Pop, (2014), "HELGA: a heterogeneous encoding lifelike genetic algorithm for population evolution modeling and simulation". Soft Computing, Vol. 18: pp.2565-2576.

[16] J. Zhang, H. Chung, and W. L. Lo, (2007), "ClusteringBased Adaptive Crossover and Mutation Probabilities for Genetic Algorithms", IEEE Transactions on Evolutionary Computation, Vol.11(3), pp. 326-335.
[17] Tomoiagă B, Chindriş M, Sumper A, Sudria-Andreu A, Villafafila-Robles R., (2013), Pareto Optimal Reconfiguration of Power Distribution Systems Using a Genetic Algorithm Based on NSGA-II. Energies, 6(3):1439-1455.

[18] Akbari, Ziarati, (2011), "A multilevel evolutionary algorithm for optimizing numerical functions" IJIEC 2 (2011): 419-430.

[19] Y.S., Zhou and L.Y. Lai, (2000), Optimal Design for Fuzzy Controllers by Genetic Algorithms. IEEE Trans. On Industry Application, Vol. 36, No. 1, pp. 93 - 97.

[20] D. E. Grzechca, (2015), Construction of an Expert System Based on Fuzzy Logic for Diagnosis of Analog Electronic Circuits, Intl Journal Of Electronics And Telecommunications, Vol. 61(1), pp. 77-82. 